\title{
Vacuum ultraviolet photoionization study of gas phase vitamins $A$ and B1 using aerosol thermodesorption and synchrotron radiation
}

Héloïse Dossmann, ${ }^{1}$ Adrián Schwarzenberg, ${ }^{1}$ Denis Lesage, ${ }^{1}$ Marie Pérot-Taillandier, ${ }^{1}$ Carlos Afonso, ${ }^{2}$ Barbara Cunha de Miranda, ${ }^{3}$ Gustavo A. Garcia ${ }^{3}$

${ }^{1}$ Université Pierre et Marie Curie, CSOB/IPCM UMR 8232, 4, Place Jussieu BP 45, 75252 Paris Cedex 05, France.

${ }^{2}$ Normandie Univ, COBRA UMR 6014 et FR 3038 ; Univ. Rouen ; INSA Rouen ; CNRS, IRCOF, 1 rue Tesnière, 76821 Mont-Saint-Aignan Cedex, France, France.

${ }^{3}$ Synchrotron SOLEIL, L'Orme des Merisiers, St Aubin, BP 48, 91192 Gif-sur-Yvette Cedex, France.

\begin{abstract}
:
Gas-phase studies of biomolecules are often difficult to initiate because of the thermolability of these systems. Such studies are nevertheless important to determine fundamental intrinsic properties of the molecules. Here we present the valence shell photoionization of gas-phase vitamins A and B1 close to their ionization threshold. The study was performed by means of an aerosol thermodesorption source coupled to an electron/ion coincidence spectrometer and synchrotron radiation (SOLEIL facility, France). Ion yield curves were recorded for both molecules over a few eV energy range and the threshold photoelectron spectrum was also obtained for vitamin A. Some fundamental properties were extracted for both ions such as adiabatic and the three first vertical ionization energies of retinol $\left(\mathrm{IE}_{\mathrm{ad} .}=6.8 \pm 0.2 \mathrm{eV}\right.$ and $\mathrm{IE}_{\mathrm{vert}}$. $=7.4,8.3$ and $9.3 \mathrm{eV}$ ) and dissociation appearance energies for the main fragment ions of vitamin B1. Analysis of the data was supported by ab initio calculations which show a very good agreement with the experimental observations.
\end{abstract}

\section{KEYWORDS:}

Gas phase; Synchrotron; Vitamin; Photoionization; Ionization energy; Appearance energy; Aerosol thermodesorption

\section{Introduction}

Gas phase studies of biomolecules provide a complementary alternative to the direct, condensed phase approach. ${ }^{1}$ Their electronic and geometric structures in their free form can be experimentally extracted and interpreted with high level ab initio models that cannot currently be extrapolated to the condensed matter due to the added complexity and number of intra- 
molecular forces. The intrinsic physico-chemical properties of these isolated systems might help understand their in-vivo biological functions within a bottom-top or pyramidal logic. Such studies are nevertheless very difficult to initiate because biomolecules are generally thermally fragile and their vaporization using classical methods often leads to their degradation. As a consequence of this experimental challenge, there is a dramatic lack of fundamental electronic properties for many important biomolecules such as ionization energies, electron affinities, etc... In this work, we have taken advantage of a relatively recent technique to bring intact neutral thermolabile vitamins into the gas phase using aerosol thermodesorption. ${ }^{2}$ We have then studied the vacuum ultraviolet (VUV) spectroscopy of vitamin A (retinol) and B1 (thiamine) (Scheme 1), which are typical systems to illustrate the amount and type of information we can collect on gas-phase biomolecules applying this vaporization technique. Only one HeI photoelectron spectroscopic study is indeed reported on vitamin $\mathrm{A}^{3}$ and, to the best of our knowledge, vitamin B1 has never been the subject of gas-phase experiments. Both molecules are nevertheless playing an essential role in many body functions such as vision, growth, reproduction for retinol and nervous system and muscle functioning for thiamine. ${ }^{4}$ Although they both have been identified for a long time (around 100 years), ${ }^{5}$ much still remains to be understood about their properties and biological functions. To this end, gas-phase experiments might yield some fundamental insights.

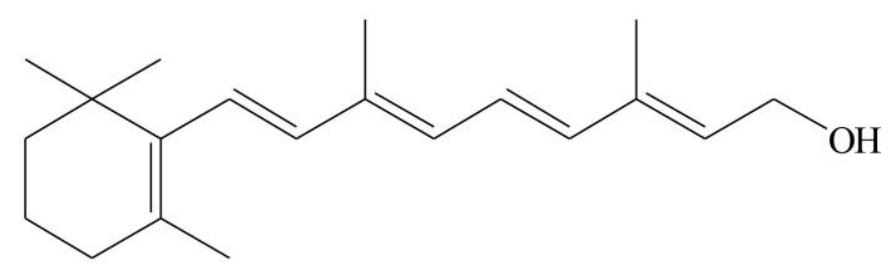

Vitamin A

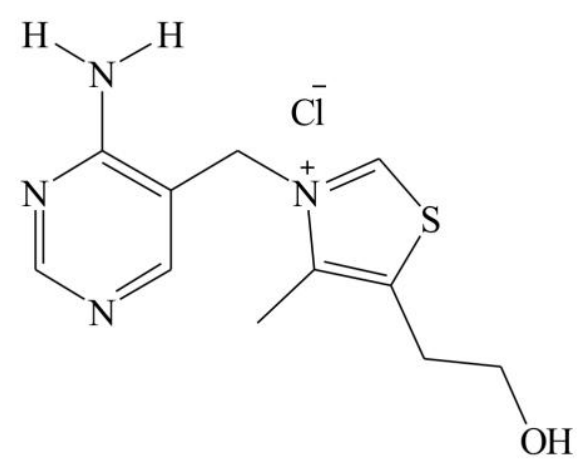

Vitamin B1

Scheme 1. Structure of vitamins A and B1. 
Furthermore, determination of thermochemical properties such as ionization energies and fragment appearance energies will be useful to the field of radiation damage.

In this work we present the threshold photoelectron spectrum of vitamin $A$ and its interpretation by means of molecular electronic structure. The ion yields for vitamin B1 and its fragments showing their relative abundance according to the photon energy are also given, from which ionization and fragment appearance energies are extracted. Ab initio calculations have been performed in order to interpret the experimental results.

\section{Experimental Method}

Experiments were carried out on the DESIRS beamline at the SOLEIL synchrotron facility in St. Aubin (France). ${ }^{6}$ This undulator-based VUV-beamline is equipped with a $6.65 \mathrm{~m}$ normal incidence monochromator proposing several gratings depending on the required flux/resolution compromise. We have chosen a $200 \mathrm{gr} / \mathrm{mm}$ grating which offers a photon flux in the $10^{12}-10^{13}$ $\mathrm{ph} / \mathrm{s}$ range with a moderate energy resolution. The slits were set to deliver a photon energy bandwidth $10 \mathrm{meV}$ at $9 \mathrm{eV}$, and the second order light was removed with an upstream gas filter filled with $\mathrm{Xe}^{7}$ The Rydberg absorption lines of $\mathrm{Xe}^{8}$ in the energy range considered yielded the absolute energy calibration with a precision better than $4 \mathrm{meV}$.

All chemicals were obtained from Sigma Aldrich and solvents were of analytical grade. Solutions of vitamins with a concentration of $0.5 \mathrm{~g} / \mathrm{L}$ were prepared in a mixture of water/ethanol 1:2 (vitamin A) and in pure water (vitamin B1). ). All solvents were of analytical grade and ultrapure water (18 $\mathrm{M} \Omega$ resistivity) was produced by a MilliQ apparatus (Millipore). The sample concentration, as well as the stoichiometry and nature of the solvent, were optimized off-line by measuring the aerosol concentration as a function of particle diameter with a scanning mobility particle sizer (SMPS, TSI model 3936L72-N). In the case of vitamin A, special precautions were taken to avoid degradation: i) the solution was prepared under $\mathrm{Ar}$ and stored in a polystyrene box; ii) during operation, the solution was placed inside a glass container covered with ice and aluminum foil.

The aerosol thermodesorption setup has been described in detail elsewhere. ${ }^{2 a}$ Briefly, the solutions are atomized and the resulting nanoparticles are dried and focused into the ionization source by means of an aerodynamic lens. They are subsequently vaporized by collision against a hot porous tungsten rod with a conically shaped tip, located $1 \mathrm{~mm}$ from the ionization region. The tungsten tip was heated at temperatures of 150 and $250{ }^{\circ} \mathrm{C}$ for the vitamins $\mathrm{A}$ and $\mathrm{B} 1$, respectively. 
The plume of intact neutral monomers is then ionized by the VUV synchrotron radiation at the center of the DELICIOUS III $^{9}$ electron/ion coincidence spectrometer, which consists of an electron velocity map imaging (VMI) device, with an ion imaging time of flight (TOF) mass spectrometer. DELICIOUS III provides mass-filtered photoelectron images, so that the electronic structure can be recorded for all the cations present in the TOF, simultaneously. This can be done at fixed photon energy, or varying the photon energy and selecting only ions in coincidence with threshold electrons. The latter, threshold photoelectron spectroscopy (TPES), has been chosen in this work due to its higher resolution, at the expense of longer acquisition times. For this work, a threshold electron energy resolution of $20 \mathrm{meV}$ has been chosen, with a $7 \mathrm{meV}$ photon energy step. Ion yield curves are also presented below, where no selection is made on the electron kinetic energy. However, it is important to note that with the chosen extraction field, ions correlated to fast electrons having kinetic energies $\mathrm{KE}_{\max }>3.5 \mathrm{eV}$ will be discriminated. The error bars shown in the figures have been obtained assuming an initial Poisson distribution on the image pixels, and applying standard error propagation formulae. The noise was determined with a linear fit of the baseline, then, the first point that rises above this baseline by more than its associated error bar is taken as the observed AE. The noise error and the slope of the experimental curve are contained in the given AE error bars.

\section{Theoretical Method}

All calculations were performed using the ORCA suite of programs. ${ }^{10}$ Geometry optimizations and frequency calculations were carried out with the spin-component scaled second-order Møller-Plesset perturbation theory (SCS-MP2) ${ }^{11}$ using the Def2-SVP basis set of Alhrichs and coworkers. ${ }^{12}$ To speed up calculations, resolution identity $(\mathrm{RI})^{13}$ approximation was applied in conjunction with the def2-SVP auxiliary basis set. Stationary points were characterized as minima (no imaginary frequencies) from vibrational frequency calculations. More accurate energies of the optimized structures were obtained using single-point energy (SPE) calculations with the orbital-optimized ${ }^{14}$ (OO) SCS-MP2 method with the triple-zeta Ahlrichs-type set def2TZVP. ${ }^{12,15}$ The $\mathrm{RI}^{13}$ and RIJCOSX ${ }^{16}$ approximations were employed in conjunction with the def2-TZVP auxiliary basis set. Additionally to its accuracy, this method was also chosen because it was giving the best results concerning spin contamination for open-shell species. We have indeed been facing a very large spin contamination issue by using more conventional methods (some were giving a spin operator value $\left\langle\mathrm{S}^{2}\right\rangle$ larger than $80 \%$ than the expected one (0.75 a.u. for doublet species)). With the OO-RI-SCS-MP2 method, we obtained the most 
satisfying results with a negligible spin contamination (less than $5 \%$ difference for the spin operator value).

For vitamin A, the calculated adiabatic ionization energy was corrected using a calibration established from several experimental adiabatic ionization energies found in the literature. The procedure is described in the supplementary information (SI). Furthermore, vertical ionization energies of retinol correspond to the energies of the three highest molecular orbitals of retinol obtained at the MP2/Def2-SVP level of theory.

All details of computational results are given in the supplementary information (SI).

\section{Results and Discussion}

\section{A. Photoionization of vitamin A}

Figure 1 shows TOF mass spectrum of retinol recorded at $10 \mathrm{eV}$ photon energy. One ion is observed, $m / z$ 286, corresponding to the intact retinol molecular ion $\mathbf{M}^{+\bullet}$. The Figure shows the advantage of using a soft vaporization technique such as aerosol thermodesorption, since no thermodegradation products are observed in the mass spectra. The precursor ion presents a temporal resolving power of $\mathrm{t} / \Delta \mathrm{t}=180$, which translates ${ }^{17}$ into a mass resolution - the value for which $\mathrm{M}$ and M+1 are fully separated in the TOF - of around $150 \mathrm{amu}$. Evidently, the mass resolution degrades with increasing ion kinetic energy, so that fragment ions are more difficult to resolve

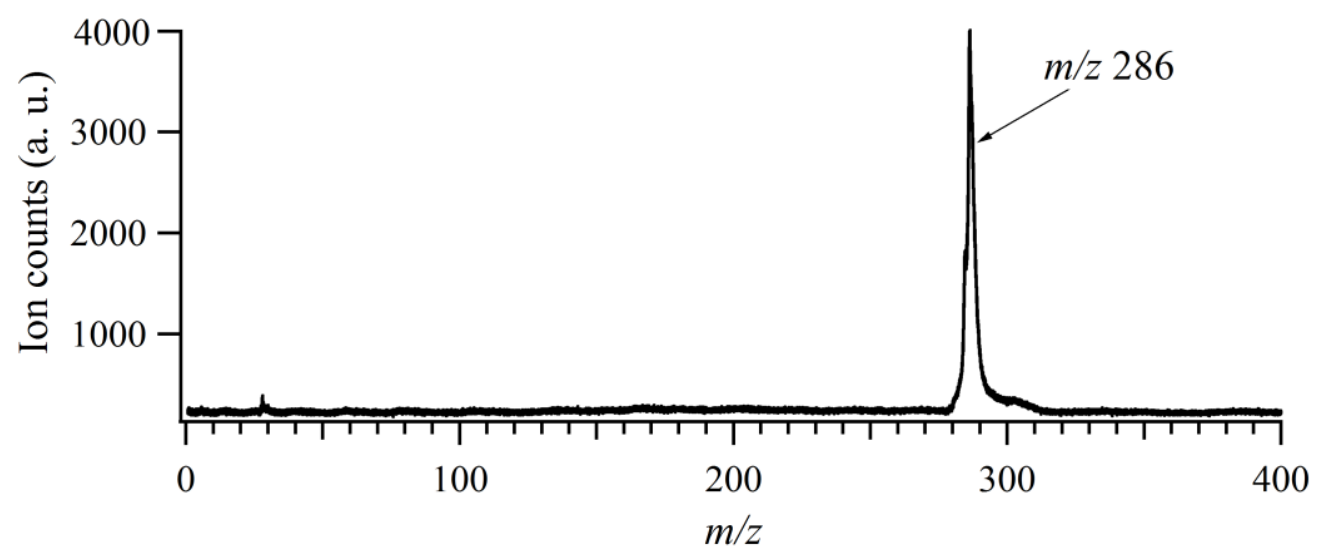

Figure 1. TOF spectra of photoionized vitamin $A$ at $10 \mathrm{eV}$. The small contribution at $\mathrm{m} / \mathrm{z} 28$ is the ionization of $\mathrm{N}_{2}$, which is the carrier gas in the aerosol setup, with the remaining second-order light.

The ion yield associated with detection of mass-selected $\mathrm{m} / \mathrm{z} 286$ ion has been recorded in the 6.3 $-10.0 \mathrm{eV}$ energy range and is shown in Figure 2a. A slow continuous onset can be observed 
in the spectrum with several slight slope changes. Since the extraction field is high enough to detect all the photoelectrons (as the energy range does not exceed $3.5 \mathrm{eV}$ above ionization threshold, see experimental part), this spectrum corresponds to the retinol total ion yield $v s$ the photon energy, providing a measurement of the relative photoionization cross section of retinol. It can further be noticed that on the spectrum in Figure $2 \mathrm{a}$, the $\mathrm{Xe} 5 \mathrm{~s}_{2} 5 \mathrm{p}_{6} \rightarrow 5 \mathrm{~s}_{2} 5 \mathrm{p}_{5}\left(2 \mathrm{P}_{3 / 2}\right) 6 \mathrm{~s}$ absorption line (coming from the gas filter) is observed at $8.437 \mathrm{eV}$ and provides an absolute energy calibration.
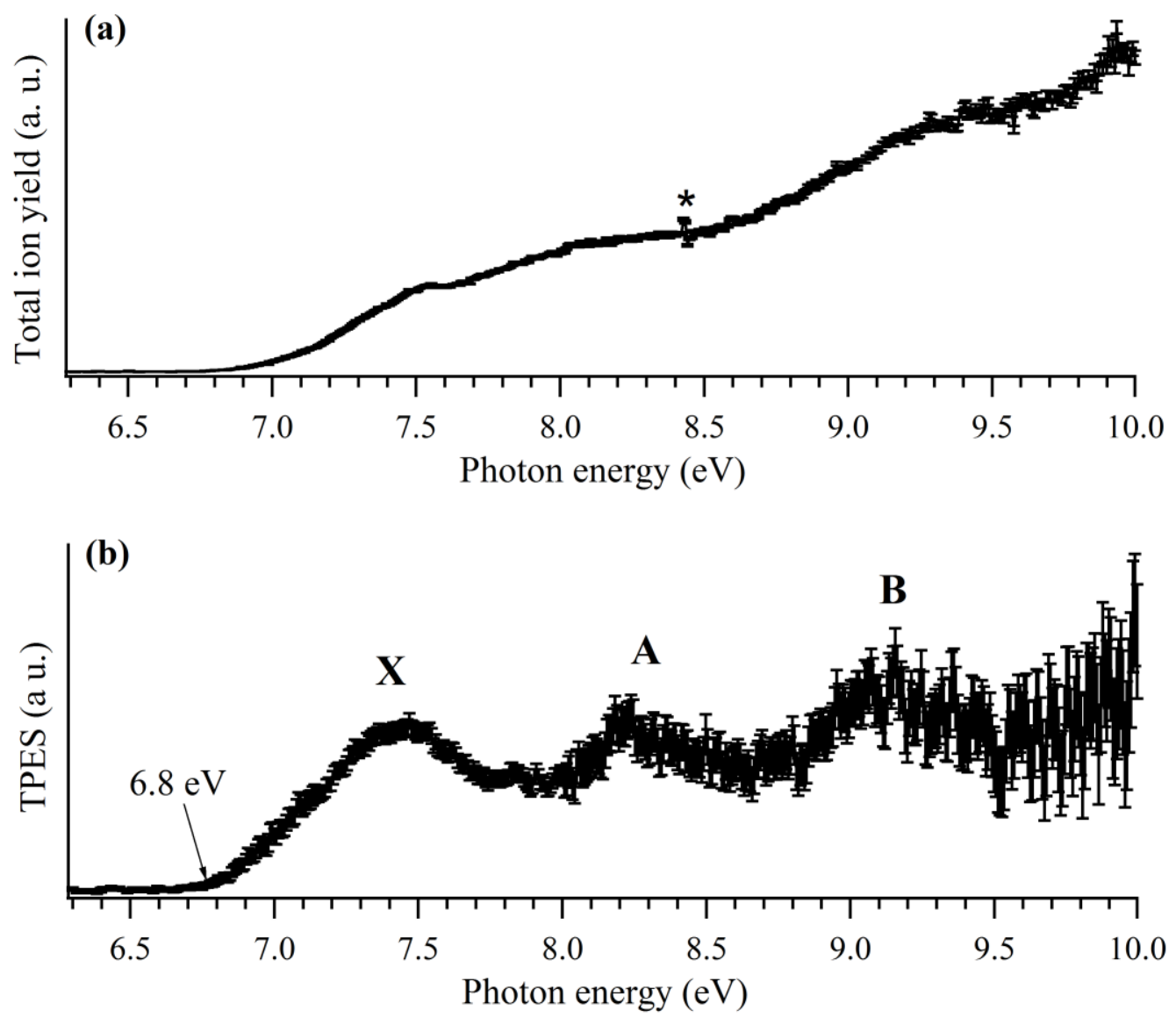

Figure 2. (a) Total ion yield of $\mathrm{m} / z 286$ ion obtained from retinol and recorded in the $6.3-10.0 \mathrm{eV}$ energy range. (b) Mass-selected TPES of $m / z 286$ ion recorded in the 6.3-10.0 eV energy range with an electron resolution of $20 \mathrm{meV}$. On Figure $2 \mathrm{a}$, the asterisk points out the $\mathrm{Xe} 5 \mathrm{~s}_{2} 5 \mathrm{p}_{6} \rightarrow 5 \mathrm{~s}_{2} 5 \mathrm{p}_{5}\left(2 \mathrm{P}_{3 / 2}\right) 6 \mathrm{~s}$ absorption line. Letters indicate the three observed bands and an estimation of the observed threshold of retinol is given on graph (b).

In Figure $2 b$, the mass-selected TPES of the $m / z, 286$ ion is presented. Three broad transition bands, termed $\mathbf{X}, \mathbf{A}$ and $\mathbf{B}$, are distinctly observed. These bands correspond to the three first vertical transitions and are centered at 7.40, 8.25 and $9.15 \mathrm{eV}$ respectively. In the work of Katsumata and Ikehata, ${ }^{3}$ a HeI photoelectron spectrum of vitamin A is presented and shows several bands in the $6-12.5 \mathrm{eV}$ energy range. The three first ones are measured at $6.95,8.15$ 
and $8.95 \mathrm{eV}$. They are by up to $0.45 \mathrm{eV}$ lower in energy than the bands we observe. We attribute this energy shift to a different internal energy of the neutral molecule. Another vaporization method (infrared heating furnace) is indeed used by Katsumata and Ikehata ${ }^{3}$ which may thus form gaseous neutral retinol with a different initial internal energy. To help analyze these data, theory has been used. According to Koopmans theorem, ${ }^{18}$ vertical ionization transitions can indeed be evaluated from the energies of highest occupied molecular orbitals (HOMOs) of retinol. Here, bands observed would thus correspond to the three first HOMOs of retinol, i. e. HOMO, HOMO-1 and HOMO-2. Results of calculations are presented in Table 1 and the structures of these three molecular orbitals in supplementary information (SI), Figure S2. Predicted MO energies differ by $\pm 0.4 \mathrm{eV}$ from our experimental vertical ionization energies. This difference is not negligible but is due to the assumption in Koopmans theorem that wavefunctions of electrons are not affected by removal of an electron which is rarely the case. It has to be noted that other theoretical methods exist to estimate more precisely vertical ionization energies but due to the relatively large size and low symmetry of retinol, we have not been able to perform them. Our calculations at least show that three bands are expected in the 6.9-9.7 eV region confirming our experimental observations.

Table 1. Experimental vertical ionization energies $\mathrm{IE}_{\mathrm{vert}}(\mathrm{eV})$ and calculated energies of the three first HOMOs of retinol $\left(-\mathrm{E}_{\mathrm{MO}}(\mathrm{eV})\right)$.

\begin{tabular}{cccl}
\hline \multirow{2}{*}{ Band } & \multirow{2}{*}{$\mathrm{IE}_{\mathrm{vert}}(\mathrm{eV})$} & \multicolumn{2}{c}{ Theory } \\
\cline { 3 - 4 } & & $-\mathrm{E}_{\mathrm{MO}}(\mathrm{eV})$ & $\mathrm{MO}$ \\
\hline $\mathbf{X}$ & 7.4 & 7.00 & HOMO \\
\hline $\mathbf{A}$ & 8.3 & 8.61 & HOMO-1 \\
\hline $\mathbf{B}$ & 9.3 & 9.64 & HOMO-2 \\
\hline
\end{tabular}

Furthermore, although the precise thermal energy of the neutral and the vibrational overlaps in the neutral $\rightarrow>$ ion vibronic transitions which define the shape of the threshold curve are not known, we can nevertheless provide an estimation of the adiabatic ionization energy, $\mathrm{IE}_{\mathrm{ad} .}=6.8$ $\pm 0.2 \mathrm{eV}$, by linear extrapolation of the TPES shown in Figure 2. Calculations provide a value of $\mathrm{IE}_{\mathrm{ad} .}=6.76 \mathrm{eV}$ which is then corrected ${ }^{\mathrm{a}}$ to give the semi-empirical value $\mathrm{IE}_{\text {ad.corr. }}=6.66 \pm$ $0.17 \mathrm{eV}$. Both values are in very good agreement with the experimental observation, supporting our choice of theoretical method. A difference between the first vertical and adiabatic ionization energies of $\sim 0.6 \mathrm{eV}$ is thus observed in the experiment, with a featureless onset,

\footnotetext{
${ }^{\text {a }}$ See supplementary information
} 
which could be the consequence of the blurring induced by the presence of different conformers and/or the large number of degrees of freedom.

The corresponding optimized structures of neutral and ionized retinol are shown in Figure 3 as well as a selection of the structural parameters which are subjected to the largest changes upon ionization. The most visible geometrical change between neutral and ion concerns the relative position of the polyene chain compared to the cyclohexene ring: whereas both parts are pretty much twisted in the neutral (dihedral angle 1-2-4-5), they appear more aligned in the cation, reinforcing the conjugation of the system.

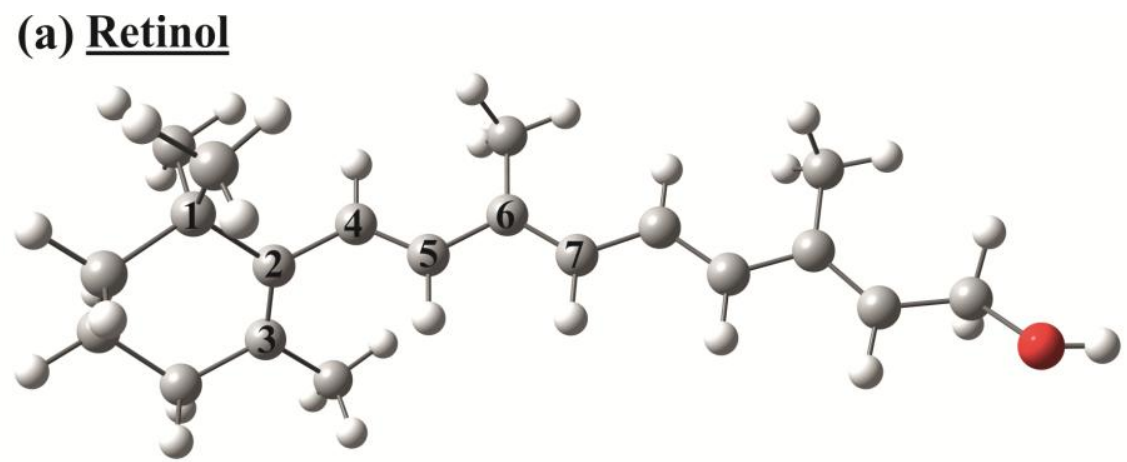

\section{(b) Ionized retinol}

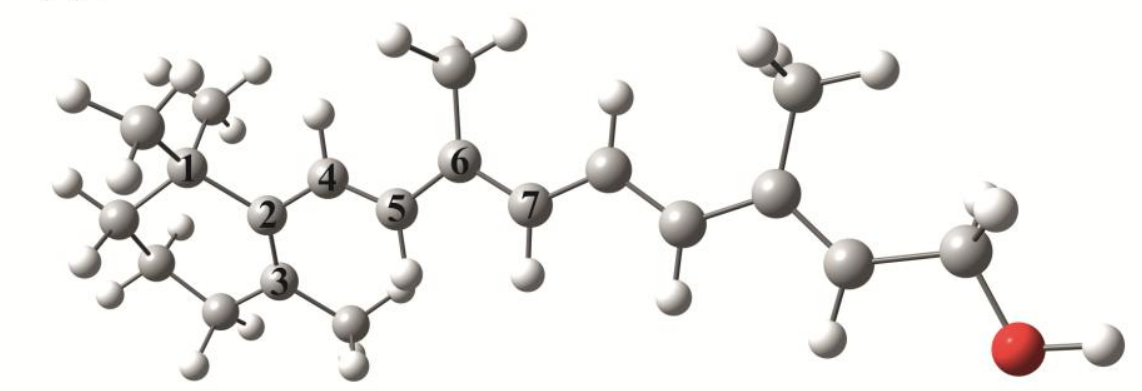

\begin{tabular}{cccccccccccc}
\cline { 2 - 11 } & \multicolumn{8}{c}{$\begin{array}{c}\text { Selected structural parameters } \\
\text { (bond lenghts in } \AA \text {, angles in degree) }\end{array}$} \\
\cline { 2 - 13 } & $\mathbf{1 - 2}$ & $\mathbf{2 - 3}$ & $\mathbf{2 - 4}$ & $\mathbf{4 - 5}$ & $\mathbf{5 - 6}$ & $\mathbf{1 - 2 - 4}$ & $\mathbf{2 - 4 - 5}$ & $\mathbf{4 - 5 - 6}$ & $\mathbf{1 - 2 - 4 - 5}$ & $\mathbf{3 - 2 - 4 - 5}$ & $\mathbf{2 - 4 - 5 - 6}$ \\
\hline (a) & 1.54 & 1.36 & 1.48 & 1.36 & 1.46 & 114.6 & 124.6 & 126.0 & 125.9 & -55.6 & -178.8 \\
(b) & 1.55 & 1.39 & 1.44 & 1.39 & 1.42 & 114.6 & 131.3 & 124.4 & 159.0 & -25.1 & 179.7 \\
\hline
\end{tabular}

Figure 3. Geometry optimized structures of (a) retinol and (b) ionized retinol. Some selected structural parameters are given in the table.

\section{B. Photoionization of vitamin B1}

Vitamin B1 has the particularity of being a salt, e.g. it is composed of two ionic parts, a cation with a $265 \mathrm{~m} / \mathrm{z}$ ratio and a $\mathrm{Cl}^{-}$anion. However, no ion signal was detected on the spectrometer when the synchrotron radiation was cut off. By turning the light on, a signal for ions and 
electrons appears proving that these ions are formed in a photoionization process. This could mean that aerosol thermodesorption is soft enough to enable the ionic bonding of vitamin B1 to remain intact in the gas-phase, although another possibility would be thermal decomposition into nonionic species with the elimination of $\mathrm{HCl}$ prior to photoionization, as has been reported before for ammonium halides. ${ }^{19}$ In Figure 4, two resulting mass spectra obtained at two different photon energies are presented. With $8.5 \mathrm{eV}$ photons, only one ion, $\mathrm{m} / \mathrm{z} 264$, is formed which corresponds to vitamin B1 having lost formally " $\mathrm{HCl}^{-\bullet "}$. Six deprotonation sites (Scheme 2) have been considered to determine the structure of this ion leading to six isomers termed $\mathbf{B 1}_{112} \mathbf{X}^{+\bullet}(\mathbf{X}=1-6)$. They all correspond to distonic ions and have energies differing by up to $1.3 \mathrm{eV}$ (Scheme 2). Further, at $12 \mathrm{eV}$ photon energy, the $\mathrm{m} / \mathrm{z}, 264$ ion has partly dissociated as shown in Figure $4 b$. Several ions indeed appear on the mass spectrum, the major ones being $\mathrm{m} / \mathrm{z}$ 233, 143, 122 and 112. A structure for these ions is proposed in Scheme 3. They correspond to the most stable structure as found by calculations (except for $\mathbf{B} \mathbf{1}_{\mathbf{1 1 2}} \mathbf{2}^{+}$, see below). Our TOF spectrum agrees well with the unique electron ionization (EI) mass spectrum that exists to our knowledge in the literature for vitamin $\mathrm{B} 1 .^{20}$ The major intensities of this spectrum are given in Table 2. The same fragments as in this work are observed in Table 2 but with different relative intensities. The EI spectrum indeed shows that the most intense fragments have low $\mathrm{m} / \mathrm{z}$ ratio which is due to the large initial deposited energy with this source, as opposed to the softer ionization achieved with tunable VUV sources. Finally, it has to be noted that we have considered only major fragments on the $12 \mathrm{eV}$ TOF spectrum in Figure $4 \mathrm{~b}$. Other low intensity fragments are also observed as $m / z 249,150,116$. We have not clearly identified the origin of these ions (i.e. if they are fragments or impurities) and because of their poor intensities, their study has not been pursued further. 

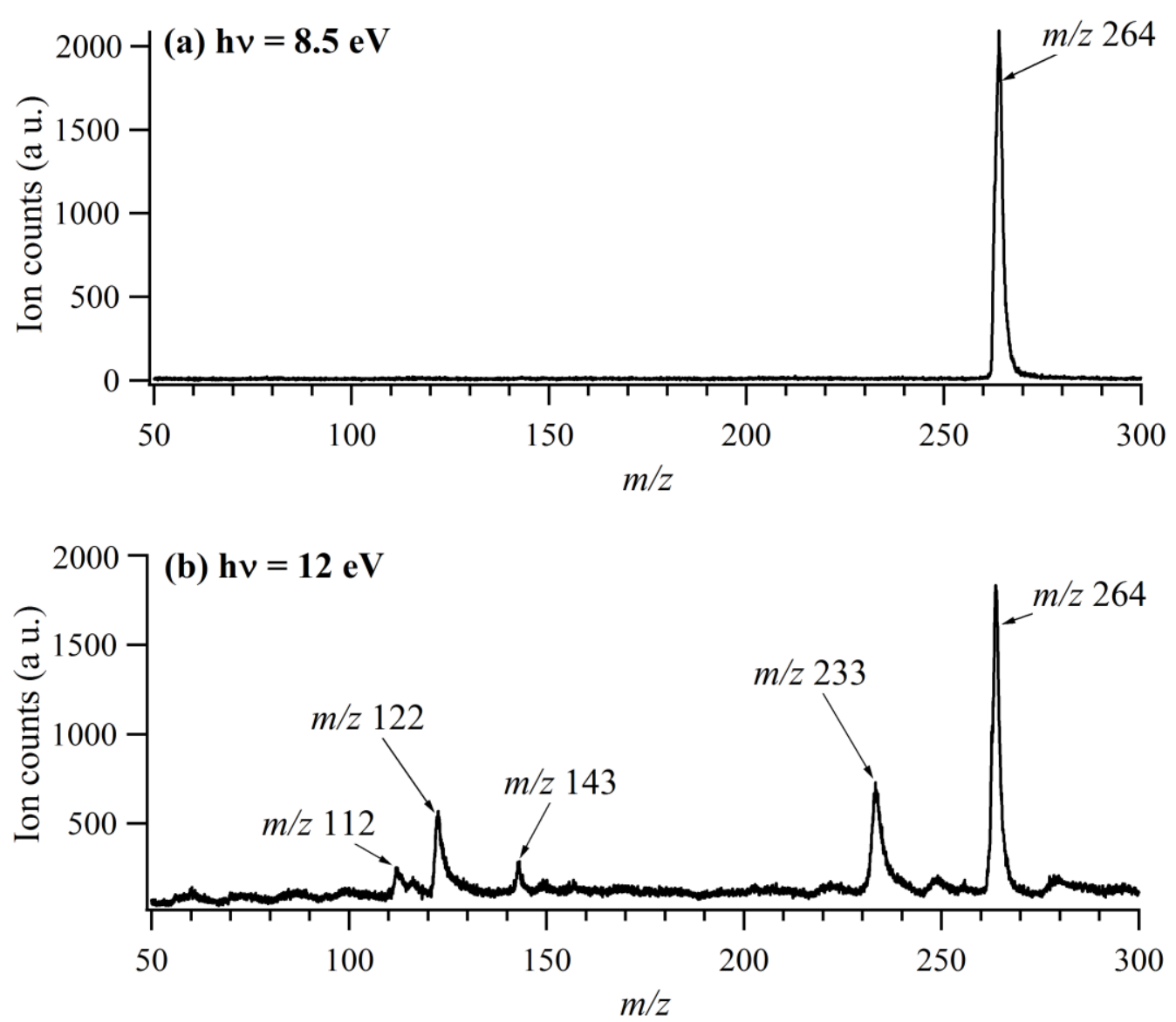

Figure 4. TOF spectra of photoionized vitamin $\mathrm{B} 1$ at (a) 8.5 and (b) $12 \mathrm{eV}$. Note that the mass resolution is about $150 \mathrm{amu}$, implying that channels like $\mathrm{H}$-loss are included alongside the parent.

Table 2. Intensities (I in \% of the base peak) of the major $\mathrm{m} / \mathrm{z}$ fragments in the electron-ionization (EI) mass spectrum of vitamin $\mathrm{B} 1 .^{20}$ $m / z(\mathrm{I})$ 43 (20), 44 (26), 53 (14), 55 (24), 57 (19), 59 (14), 69 (25), 71 (22), 81 (21), 83 (16), 85 (42), 98 (17), 112 (100), 122 (37), 143 (29), 233 (8), 264 (7) 


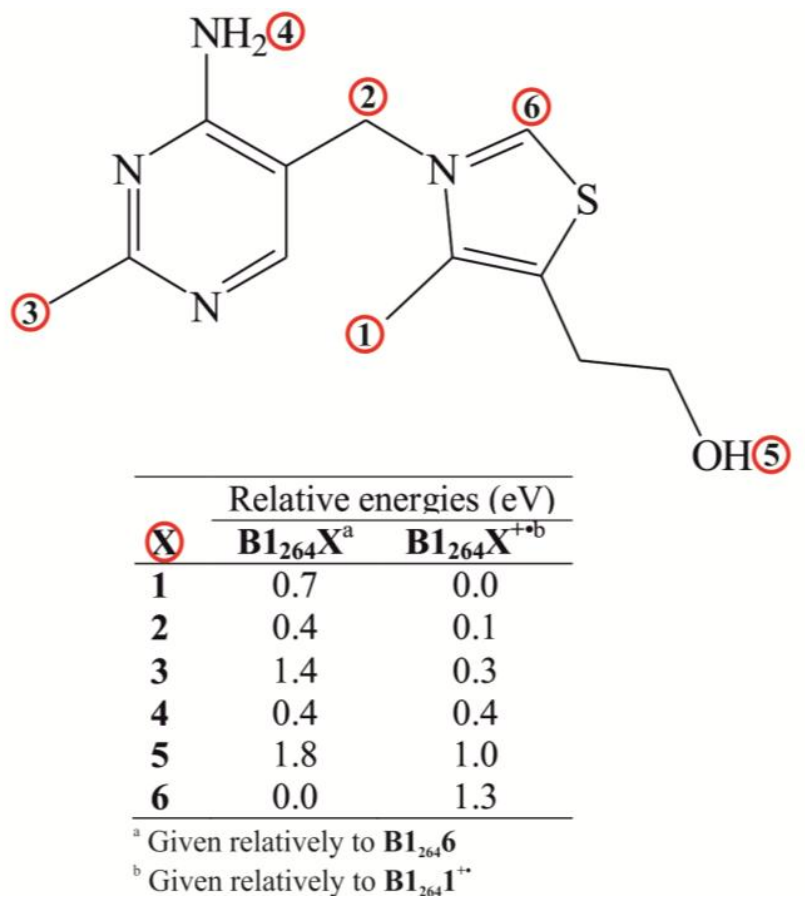

Scheme 2. Deprotonation sites (indicated by circles) on the thiamine backbone which have been considered for $\mathbf{B} 1_{264} \mathbf{X}$ and $\mathbf{B} 1_{264} \mathbf{X}^{+\bullet}(\mathbf{X}=1-6)$. The calculated relative energies are given in the table.

Ion yield curves have been registered for the $m / z, 264$ ion and its four major fragments. Results are shown in Figure 5. For each ion, its appearance energy has been evaluated (by giving the energy value of the first point going out from the noise). Hence, the $\mathrm{m} / \mathrm{z} 264$ ion is produced starting from $7.0 \mathrm{eV}$. The first fragment to be formed is the $\mathrm{m} / \mathrm{z} 233$ ion appearing $2 \mathrm{eV}$ above the $\mathrm{m} / \mathrm{z} 264$ threshold $(9.0 \mathrm{eV})$. It is followed by the $\mathrm{m} / \mathrm{z} 143$ ion which emerges at $9.4 \mathrm{eV}$. The two last fragments, $m / z 122$ and 112, appear at 9.5 and $9.8 \mathrm{eV}$, respectively. We have compared these experimental observations with appearance energies (AEs) calculations. Two hypotheses prevail to explain the formation of the $m / z 264$ ion: it can be formed either by an $\mathrm{HCl}$ loss leading to a molecule $\mathbf{B 1} \mathbf{2 6 4}_{\mathbf{2}} \mathbf{X}(\mathbf{X}=1-6$, Scheme 2) which is then photoionized or by first photoionization of thiamine followed by an $\mathrm{HCl}$ release. The corresponding required energies have been calculated and results are presented in Table 3. Direct photoionization of thiamine followed by an $\mathrm{HCl}$ elimination costs between 7.0 and $8.3 \mathrm{eV}$ depending on the $\mathrm{m} / \mathrm{z}, 264$ isomer considered. This process is more expensive than photoionization of the $\mathbf{B 1}_{\mathbf{2 6 4}} \mathbf{X}(\mathbf{X}=1-6)$ molecules (which have first lost $\mathrm{HCl}$ from thiamine) as the corresponding ionization energies are comprised between 5.1 and $6.2 \mathrm{eV}$ with an exception for $\mathbf{B 1} \mathbf{2 6 4}_{\mathbf{2 6 4}} \mathbf{6}$ which has an IE of $7.5 \mathrm{eV}$. This set of results suggests that direct photoionization of thiamine (termed further process 1 ) is necessarily occurring because the appearance energy threshold of the ion is ca. $7.0 \mathrm{eV}$ and the expected energies for photoionization of $\mathbf{B 1}_{\mathbf{2 6 4}} \mathbf{X}$ are either too low (between 5.1 and $6.2 \mathrm{eV}$ for 
$\mathbf{X}=1-5)$ or too high (7.5 eV for $\left.\mathbf{B} 1_{264} \mathbf{6}\right)$. This feature underlines the softness of the aerosol source as it shows that the ionic bond was conserved after thermodesorption. We can nevertheless not exclude the possibility that some $\mathrm{m} / \mathrm{z} 264$ ions may also originate from photoionization of $\mathbf{B} \mathbf{1}_{\mathbf{2 6 4}} \mathbf{6}$ (termed further process 2). This hypothesis will be accordingly considered for the analysis of the data below. Note that we could have confirmed or not this postulate if a signal for $\mathrm{HCl}^{+\bullet}$ would have been observed on the TOF spectrum (as $\mathrm{HCl}$ is lost from thiamine before ionization). The $\mathrm{IE}$ of $\mathrm{HCl}$ was however not included in the energy range we have studied $(\mathrm{IE}(\mathrm{HCl})=12.744 \mathrm{eV}){ }^{21}$

AEs of the observed product ions have been calculated and are presented in Table 4. Considering first the hypothesis of process 1 occurring, predicted AE for $m / z 233$ and 143 ions are in excellent agreement with experiment with resp. only 0.1 and $0.2 \mathrm{eV}$ energy difference.<smiles>C=c1sc[n+](Cc2cnc(C)nc2N)c1=C</smiles>

$\mathrm{B1}_{233} 1^{+}$
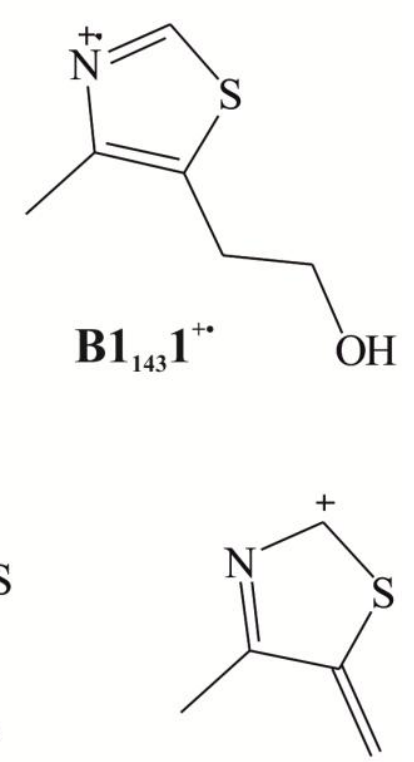

$\mathrm{B1}_{112} 2^{+}$

Scheme 3. Proposed structures for major fragment ions observed in the TOF spectrum $(12 \mathrm{eV})$ of vitamin B1.

The difference is a bit larger for $m / z$ 122: ca. $0.4 \mathrm{eV}$. It becomes very large for the last ion, $\mathrm{m} / \mathrm{z}$ 112, with $1.5 \mathrm{eV}$ difference. Assuming now ions formed via process 2, calculated AEs present more difference with experiment than with process 1 except for the $m / z, 122$ ion. Thus a difference of $0.8 \mathrm{eV}$ exists between observed and calculated value for the $m / z, 233$ ion. In the case of the $\mathrm{m} / \mathrm{z} 143$ ion, no mechanism of formation could be envisaged starting from $\mathbf{B 1}_{\mathbf{2 6 4}} \mathbf{6}^{+}$ and, for this reason, no AE value is given in Table 3. The AE for $m / z, 122$ is predicted to be 9.2 
$\mathrm{eV}$ which is only $0.3 \mathrm{eV}$ lower than the observed value. And, an $\mathrm{AE}$ of $12.1 \mathrm{eV}$ is calculated for $\mathrm{m} / \mathrm{z}, 112$ which is more than $2 \mathrm{eV}$ different from experimental measurement. Discrepancies between experiment and theory may be explained by the fact that the latter just measures the difference in the optimized energies between neutral and final fragment, neglecting dynamical effects such as the presence of transition states and barriers. Thus, large differences may forebode the presence of large fragmentation barriers. In addition, the aerosol source is known to produce neutrals with a non-negligible initial internal energy. ${ }^{22}$ The observed AES are thus expected to be shifted towards lower energies. This trend may be nevertheless counterbalanced by other factors such as i) the internal energy of the ionic fragments; ii) the presence of fragmentation barriers or of different conformers; iii) or the kinetic shift due to the fragmentation not being infinitely fast with respect to the ion residence time in the spectrometer. All these factors are expected to increase the values of observed AEs. Differences between observed and calculated AEs may thus be explained by all these features. Only a detailed knowledge of the initial internal energy of the system and of the dissociation mechanisms involved would help to correct observed AEs to obtain adiabatic AEs. And despite our efforts to check comprehensively all possible structures for fragments, it cannot be totally excluded that a lower energy structure was missed.

Table 3. Calculated appearance energies of $\mathbf{B 1}_{264} \mathbf{X}^{+\bullet}$ ions relatively to thiamine and $\mathbf{B 1}_{264} \mathbf{6}$ molecules $(\mathbf{X}=1-6)$.

\begin{tabular}{|c|c|c|}
\hline & \multicolumn{2}{|c|}{ Appearance energy $(\mathrm{eV})$} \\
\hline & thiamine & $B 1_{264} 6^{\mathrm{a}}$ \\
\hline $\mathrm{B1}_{264} \mathbf{1}^{+\bullet}$ & 7.0 & 5.5 \\
\hline$B 1_{264} 2^{+\bullet}$ & 7.1 & 5.9 \\
\hline $\mathrm{B1}_{264} 3^{+\bullet}$ & 7.3 & 5.1 \\
\hline$B 1_{264} 4^{+\bullet}$ & 7.4 & 6.2 \\
\hline$B 1_{264} 5^{+\bullet}$ & 7.9 & 5.3 \\
\hline$B 1_{264} 6^{+\bullet}$ & 8.3 & 7.5 \\
\hline
\end{tabular}

Table 4. Experimental and calculated appearance energies (in $\mathrm{eV}$ ) of the different product ions observed during photoionization of vitamin B1.

\begin{tabular}{|c|c|c|c|}
\hline & \multicolumn{3}{|c|}{ Appearance energies (eV) } \\
\hline & \multirow{2}{*}{ experimental } & \multicolumn{2}{|c|}{ calculated and relatively to } \\
\hline & & thiamine & $B 1_{264} 6$ \\
\hline$m / z 233$ & 9.0 & 9.1 & 9.9 \\
\hline$m / z 143$ & 9.4 & 9.6 & - \\
\hline $\mathrm{m} / \mathrm{z} 122$ & 9.5 & 9.1 & 9.2 \\
\hline
\end{tabular}


$\frac{m / z 112}{{ }^{a} \text { Obtained for formation of } \mathbf{B 1}_{\mathbf{1 1 2}} \mathbf{1}^{+} \text {. In the case of } \mathbf{B 1}_{\mathbf{1 1 2}} \mathbf{2}^{+} \text {, the calculated }}$ relative energy is $11.4 \mathrm{eV}$.

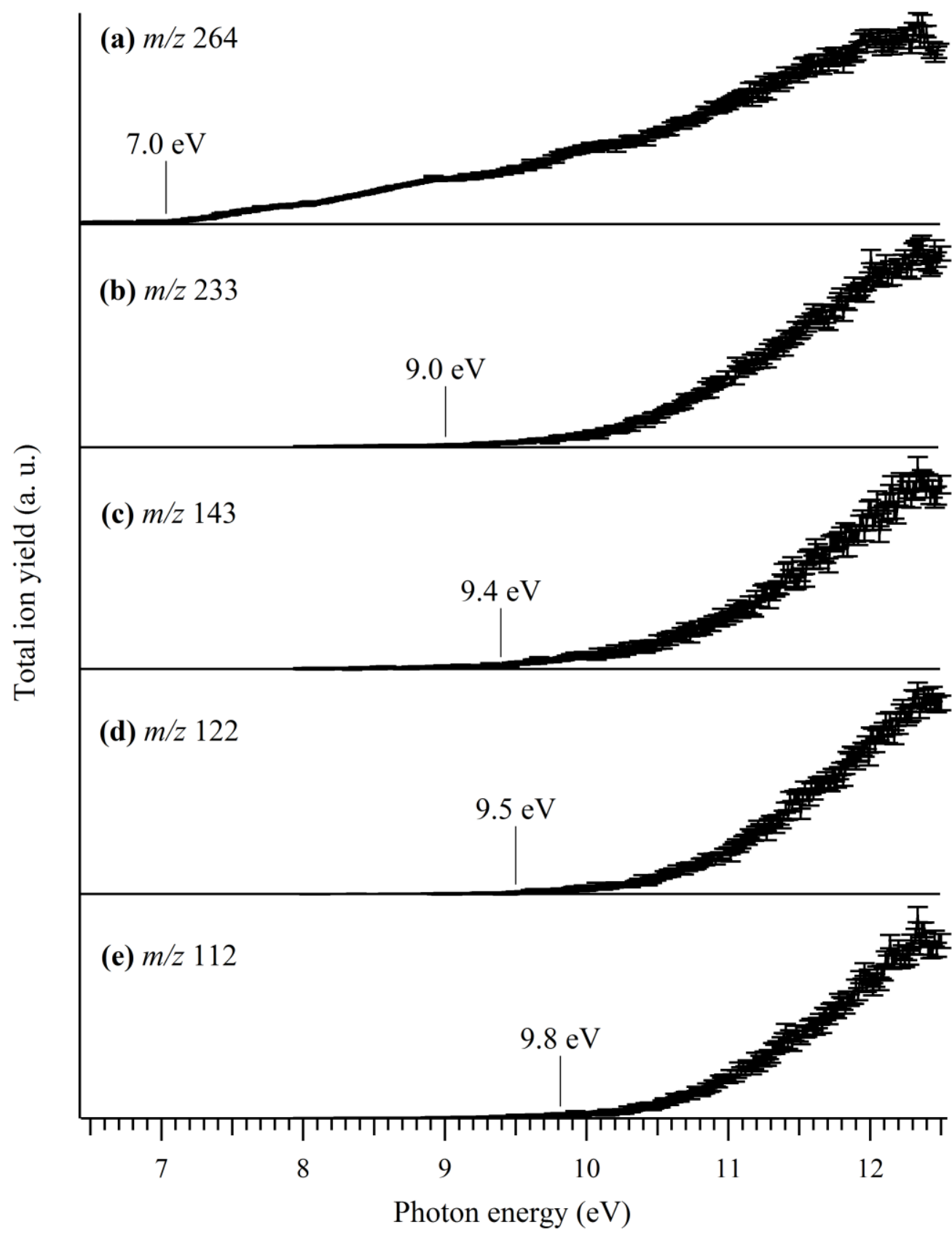

Figure 5. Total ion yields of $\mathrm{m} / \mathrm{z}$ (a) 264, (b) 233, (c) 143, (d) 122 and (e) 112 ions recorded in the (a) and (c) 6.5-12.5 eV energy range and (b), (d) and (e) 8-12.5 eV energy range. On each curve, the observed appearance energy is indicated. It corresponds to the first value that comes out of the baseline within the given error bars.

Mechanisms of formation of the major fragments of vitamin B1 have also been considered. For $\mathrm{m} / \mathrm{z} 233$ and 122 ions, they can be envisaged as one-step reaction driven either by the charge or 
the radical starting from $\mathbf{B} \mathbf{1}_{\mathbf{2 6 4}} \mathbf{1}^{+\bullet}$. Thus, a homolytic bond cleavage leads to $\mathbf{B} \mathbf{1}_{\mathbf{2 3 3}} \mathbf{1}^{+}$via a ${ }^{\circ} \mathrm{CH}_{2} \mathrm{OH}$ elimination (Scheme 4a) whereas charge-driven dissociation gives $\mathbf{B 1}_{\mathbf{1 2 2}} \mathbf{1}^{+}$and a methyl-thiazoleethanol radical (Scheme 4c). Formation of $\mathbf{B 1}_{\mathbf{1 4 3}} \mathbf{1}^{\mathbf{*}}$ is rationalized by considering the presence of the $\mathbf{B} \mathbf{1}_{\mathbf{2 6 4}} \mathbf{3}^{+\boldsymbol{\bullet}}$ isomer. However, recent experiments with the same aerosol source place the internal energy not far from the thermodesorber thermal energy, ${ }^{23}$ so that in this case we should expect internal energies of the order of $530 \mathrm{~K}(45 \mathrm{meV})$. This would lead to a Boltzmann population of only 1000:1 for the $\mathbf{B} \mathbf{1}_{\mathbf{2 6 4}} \mathbf{1}^{+\boldsymbol{\bullet}}: \mathbf{B 1}_{\mathbf{2 6 4}} \mathbf{3}^{+\boldsymbol{\bullet}}$ ratio so other alternatives to thermal isomerization after ionization need to be considered. Therefore, we suggest the presence of a transition state leading to the $\mathbf{B 1}_{\mathbf{2 6 4}}{ }^{+\bullet}$ isomers through the loss of $\mathrm{HCl}$, so that the isomer ratio is controlled dynamically rather than thermally. From the $\mathbf{B} \mathbf{2}_{\mathbf{2 6 4}} \mathbf{3}^{+\bullet}$ precursor, loss of a pyrimidinimine neutral leads to $\mathbf{B} \mathbf{1}_{\mathbf{1 4 3}} \mathbf{1}^{+\bullet}$ via a homolytic bond cleavage (Scheme 4b).

Last, a consecutive mechanism has to be formulated to explain formation of $m / z$ 112. Starting from $\mathbf{B 1}_{233} \mathbf{1}^{+}$ion, a hydride transfer (Scheme 5a) is leading to the most stable isomeric form of $m / z$ 112, $\mathbf{B} 1_{112} \mathbf{1}^{+}$, or from $\mathbf{B} 1_{143}{ }^{+\bullet}$, a ${ }^{\cdot} \mathrm{CH}_{2} \mathrm{OH}$ elimination gives the less stable $\mathbf{B} 1_{112} 2^{+}$ion (Scheme 5b). Note that formation pathways of $\mathrm{m} / \mathrm{z} 233, \mathrm{~m} / \mathrm{z}, 122$ and 112 ions have also been envisaged assuming they originate from $\mathbf{B 1}_{\mathbf{2 6 4}} \mathbf{6}^{+\bullet}$ (process 2 ). The corresponding mechanisms are presented in the supplementary information (Figure S3). 

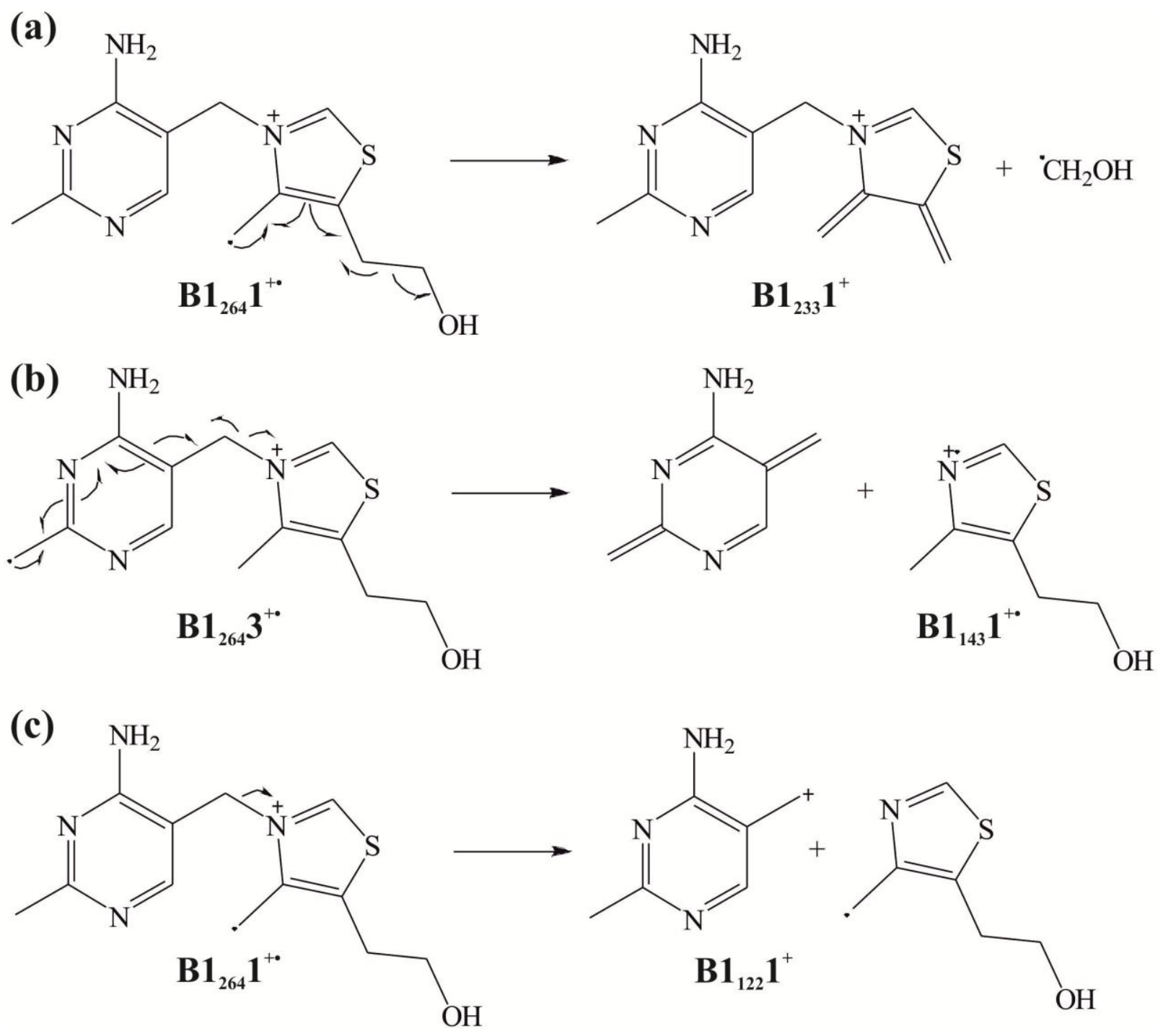

Scheme 4. Proposed mechanisms for formation of ions (a) $\mathbf{B 1}_{233} \mathbf{1}^{+}$, (b) $\mathbf{B 1}_{143} \mathbf{1}^{+\bullet}$ and (c) $\mathbf{B 1}_{\mathbf{1 2 2}} \mathbf{1}^{+}$.<smiles></smiles>

$$
\mathrm{B1}_{233} \mathbf{1}^{+}
$$

(b)

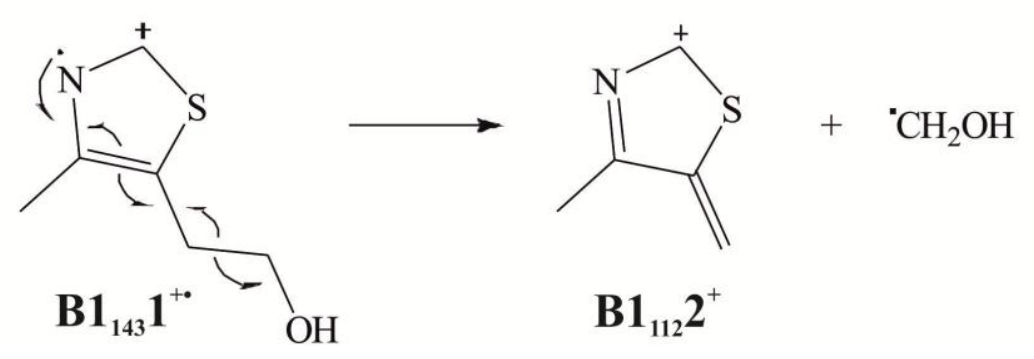


Scheme 5. Proposed mechanisms for formation of $\mathbf{B} \mathbf{1}_{112}{ }^{+}$.

Finally, the breakdown diagram of photoionized vitamin B1 has been derived from the ion yield curves and is plotted in Figure 6. This graph shows the relative abundance of each ion according to the photon energy. Three fragments, e.g. $\mathrm{m} / \mathrm{z}, 233, \mathrm{~m} / \mathrm{z} 122$ and $\mathrm{m} / \mathrm{z} 112$, appear to have similar intensities whereas the signal for $m / z, 143$ is clearly lower. The parent falls below the $50 \%$ mark for photon energies above $10.7 \mathrm{eV}$.

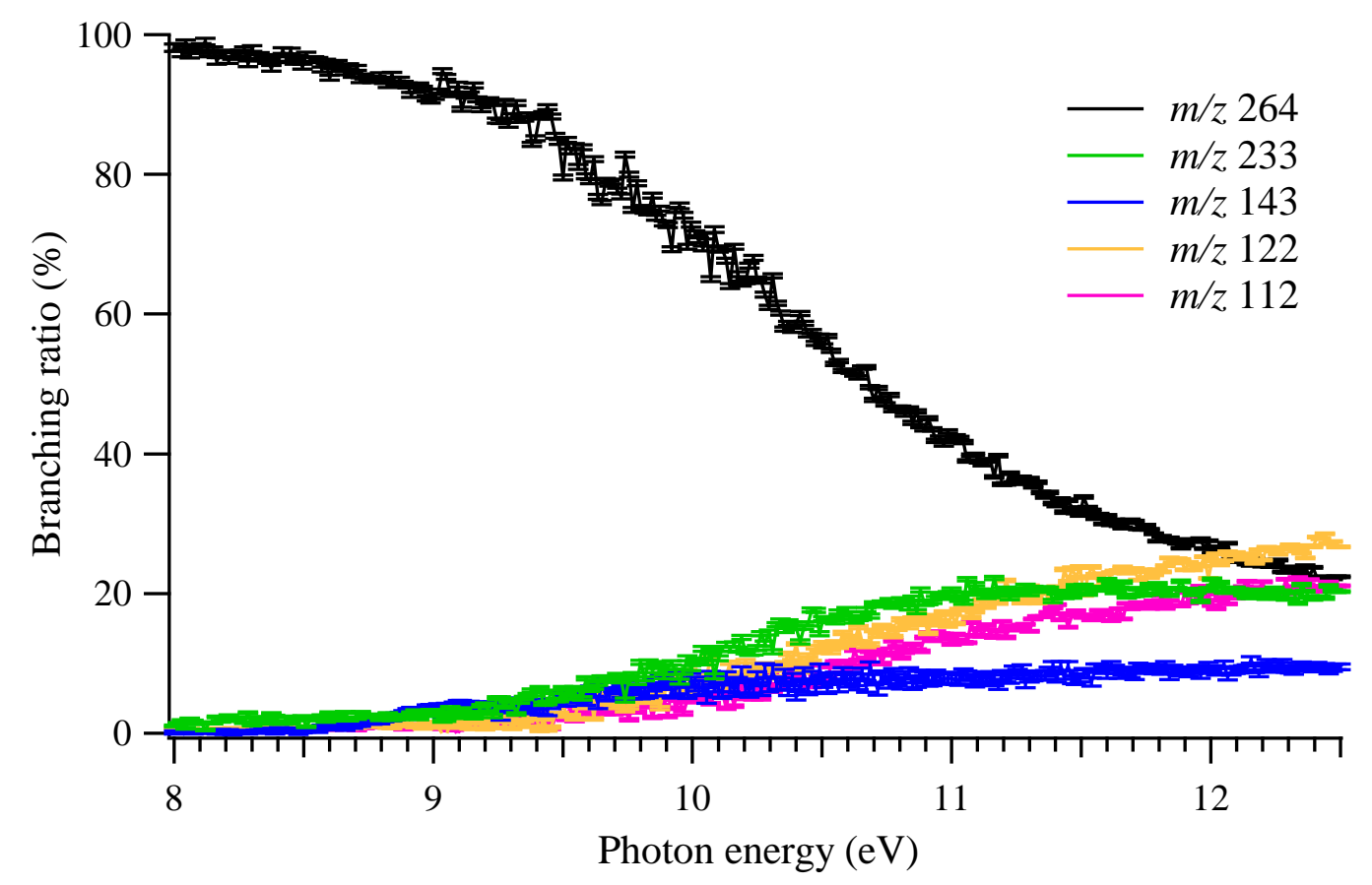

Figure 6. Breakdown diagram for dissociative photoionization of vitamin B1.

\section{CONCLUSIONS}

This paper presents a combined experimental and theoretical work on the valence shell photoionization of two vitamins, A and B1. The experimental part was made possible with the use of an aerosol thermodesorption source enabling the soft vaporization of these thermolabile molecules, and the use of electron/ion coincidences. The mass-selected TPES of vitamin A was obtained and led to an evaluation of the adiabatic and of the three first vertical ionization energies of vitamin $\mathrm{A}\left(\mathrm{IE}_{\mathrm{ad}}=6.8 \pm 0.2 \mathrm{eV}\right.$ and $\mathrm{IE}_{\text {vert. }}=7.4,8.3$ and $\left.9.3 \mathrm{eV}\right)$. The very good agreement with the calculated ionization energies (within few hundreds of meV) bestows confidence on the calculated geometries of the neutral and ionic free forms presented in this work.

In the case of vitamin B1, we presented data on the dissociative photoionization recorded up to $4.5 \mathrm{eV}$ above its first ionization threshold. The first signal from Vitamin B1 is observed at 7.0 
$\mathrm{eV}$ and a number of fragments have been identified. Comparison of the experimental appearance energies with theoretical calculations yields the fragment structures and dissociation mechanisms for most of the dissociation channels observed.

\section{ACKNOWLEDGEMENTS}

The authors acknowledge the technical support of Jean-François Gil (DESIRS) on the SAPHIRS setup and are grateful to the general staff of SOLEIL for smoothly running the facility. GAG thanks Alexandre Giuliani for helpful discussions. Finally, a huge thanks goes to Dr. Etienne Derat (UPMC) for introducing us to the ORCA software, for all his tips and advices and for giving access to calculation cluster.

\section{REFERENCES}

1. Schermann, J. P., Spectroscopy and Modeling of Biomolecular Building Blocks. Elsevier Science Ltd: 2008.

2. (a) Gaie-Levrel, F.; Garcia, G. A.; Schwell, M.; Nahon, L., VUV state-selected photoionization of thermally-desorbed biomolecules by coupling an aerosol source to an imaging photoelectron/photoion coincidence spectrometer: case of the amino acids tryptophan and phenylalanine. Phys. Chem. Chem. Phys. 2011, 13 (15), 7024-7036; (b) Fang, W.; Lei, G.; Shan, X.; Liu, F.; Wang, Z.; Sheng, L., A VUV photoionization organic aerosol mass spectrometric study with synchrotron radiation J. Electron. Spectrosc. Relat. Phenom. 2011, 184, 129-133; (c) Mysak, E. R.; Wilson, K. R.; Jimenez-Cruz, M.; Ahmed, M.; Baer, T., Synchrotron radiation based aerosol time-of-flight mass spectrometry for organic constituents. Anal. Chem. 2005, 77, 5953-5960.

3. Katsumata, S.; Ikehata, N., HeI photoelectron spectroscopic study of vitamin A and its derivatives. J. Electron. Spectrosc. Relat. Phenom. 2000, 107 (2), 139-145.

4. (a) Chung, S. S. W.; Wang, X.; Wolgemuth, D. J., Male sterility in mice lacking retinoic acid receptor $\alpha$ involves specific abnormalities in spermiogenesis. Differentiation 2005, 73 (4), 188-198; (b) McCaffery, P. J.; Adams, J.; Maden, M.; Rosa-Molinar, E., Too much of a good thing: retinoic acid as an endogenous regulator of neural differentiation and exogenous teratogen. Eur. J. Neurosci. 2003, 18 (3), 457-472; (c) Takahashi, N., Vitamin A in Health. Journal of Health Science 2010, 56 (2), 144-153; (d) McGraw, C. P.; Metcalf, D. L., Effects of thiamine, ascorbic acid, and alpha-tocopherol on neuronal and muscular function. J. Appl. Nutr. 1975, 27 (1), 51-63; (e) Murakami, Y.; Kikuchi, J.-i.; Hisaeda, Y.; Hayashida, O., Artificial Enzymes. Chem. Rev. 1996, 96 (2), 721-758. 
5. (a) McCollum, E. V.; Davis, M. J., The necessity of certain lipids during growth. $J$. Biol. Chem. 1913, 15, 167-175; (b) Funk, C., Importance of pyridine compounds in nutrition in relation to the vitamin B1 problem. Z. Vitaminforsch. 1939, 8, 326.

6. (a) DESIRS

http://www.synchrotronsoleil.fr/portal/page/portal/Recherche/LignesLumiere/DESIRS; (b) Nahon, L.; Oliveira, N. d.; Garcia, G. A.; Gil, J.-F.; Pilette, B.; Marcouillé, O.; Lagarde, B.; Polack, F., DESIRS : a stateof-the-art VUV beamline featuring high resolution and variable polarization for spectroscopy and dichroism at SOLEIL J. Synchrotron Radiat. 2012, 59 (4), 508-520.

7. Mercier, B.; Compin, M.; Prevost, C.; Bellec, G.; Thissen, R.; Dutuit, O.; Nahon, L., Experimental and theoretical study of a differentially pumped absorption gas cell used as a low energy-pass filter in the vacuum ultraviolet photon energy range. J. Vac. Sci. Technol. A 2000, $18,2533-2541$.

8. Chan, W. F.; Cooper, G.; Guo, X.; Burton, G. R.; Brion, C. E., Absolute optical oscillator strengths for the electronic excitation of atoms at high resolution. III. The photoabsorption of argon, krypton, and xenon. Phys. Rev. A 1992, 46 (1), 149-171.

9. Garcia, G. A.; Cunha de Miranda, B. K.; Tia, M.; Daly, S.; Nahon, L., DELICIOUS III: A multipurpose double imaging particle coincidence spectrometer for gas phase vacuum ultraviolet photodynamics studies. Rev. Sci. Instrum. 2013, 84 (5), 053112-053111.

10. Neese, F., ORCA - An ab Initio, Density Functional and Semiempirical Program Package, version 2.9, Mülheim and der Ruhr, 2013.

11. (a) Møller, C.; Plesset, M. S., Note on an Approximation Treatment for Many-Electron Systems. Phys. Rev. 1934, 46, 618-622; (b) Grimme, S., Improved second-order Møller-Plesset perturbation theory by separate scaling of parallel- and antiparallel-spin pair correlation energies. J. Chem. Phys. 2003, 118, 9095-9102.

12. Schäfer, A.; Horn, H.; Ahlrichs, R., Fully optimized contracted Gaussian basis sets for atoms Li to Kr. J. Chem. Phys. 1992, 97 (4), 2571-2577.

13. Eichkorn, K.; Weigend, F.; Treutler, O.; Ahlrichs, R., Auxiliary basis sets for main row atoms and transition metals and their use to approximate Coulomb potentials. Theor. Chem. Acc. 1997, 97, 119-124.

14. Kossmann, S.; Neese, F., Correlated ab Initio Spin Densities for Larger Molecules: Orbital-Optimized Spin-Component-Scaled MP2 Method. J. Phys. Chem. A 2010, 114, 1176811781. 
15. Weigend, F.; Ahlrichs, R., Balanced basis sets of split valence, triple zeta valence and quadruple zeta valence quality for $\mathrm{H}$ to Rn: Design and assessment of accuracy. Phys. Chem. Chem. Phys. 2005, 7 (18), 3297-3305.

16. Neese, F.; Wennmohs, F.; Hansen, A.; U., B., Efficient, approximate and parallel Hartree-Fock and hybrid DFT calculations. A 'chain-of-spheres' algorithm for the HartreeFock exchange. Chem. Phys. 2009, 356, 98-109.

17. Wiley, W. C.; Maclaren, I. H., Time-Of-Flight Mass Spectrometer with Improved Resolution. Rev. Sci. Instrum. 1955, 26 (12), 1150-1157.

18. Koopmans, T., Über die Zuordnung von Wellenfunktionen und Eigenwerten zu den Einzelnen Elektronen Eines Atoms. Physica 1934, 1 (1-6), 104-113.

19. Veith, H. J., Mass spectrometry of ammonium and iminium salts. Mass Spectrom. Rev. 1983, 2, 419-446.

20. obtained from SciFinder, Wiley Subscription Services, Inc. (US), spectrum CAS2009 1 163140.

21. Linstrom, P. J.; Mallard, W. G. NIST Chemistry WebBook, NIST Standard Reference Database Number 69, National Institute of Standards and Technology, Gaithersburg MD, 20899

(accessed october 2014).

22. (a) Touboul, D.; Gaie-Levrel, F.; Garcia, G. A.; Nahon, L.; Poisson, L.; Schwell, M.; Hochlaf, M., VUV photoionization of gas phase adenine and cytosine: A comparison between oven and aerosol vaporization. J. Chem. Phys. 2013, 138 (9), 094203; (b) Wilson, K. R.; Jimenez-Cruz, M.; Nicolas, C.; Belau, L.; Leone, S. R.; Ahmed, M., Thermal Vaporization of Biological Nanoparticles: Fragment-Free Vacuum Ultraviolet Photoionization Mass Spectra of Tryptophan, Phenylalanine-Glycine-Glycine, and $\beta$-Carotene. J. Phys. Chem. A 2005, 110 (6), 2106-2113.

23. Tia, M.; Miranda, B. C. d.; Daly, S.; Gaie-Levrel, F.; Garcia, G. A.; Nahon, L.; Powis, I., VUV Photodynamics and Chiral Asymmetry in the Photoionization of Gas Phase Alanine Enantiomers. J. Phys. Chem. A 2014, 118, 2765-2779. 